\title{
Development of Computer-Assisted Radial Head Replacement
}

\author{
Rebecca A. Stacpoole ${ }^{1}$, Louis M. Ferreira ${ }^{1}$, Graham J.W. King ${ }^{1,2,3}$, \\ and James A. Johnson ${ }^{1,2,3,4}$ \\ ${ }^{1}$ Hand and Upper Limb Centre, Bioengineering Laboratory, \\ St. Joseph's Health Care London, \\ 268 Grosvenor St., London, ON, Canada, N6A 4L6 \\ ${ }^{2}$ Department of Medical Biophysics \\ ${ }^{3}$ Department of Surgery, \\ ${ }^{4}$ Department of Mechanical and Materials Engineering, \\ University of Western Ontario, London, ON, Canada \\ jajohnso@uwo.ca
}

\begin{abstract}
In the cases of unreconstructible comminuted radial head fractures with associated soft tissue injuries, radial head implant replacement (or arthroplasty) is indicated. Current implant designs are axisymmetric which may prevent optimal performance, as the native radial head is offset with respect to the radial neck. To study the effect of implant position on joint tracking and kinematics, a computer-assisted implant placement procedure was developed for use in vitro with a prototype adjustable radial head implant. While the implant head was rotated and translated, the head position could be visualized and described in real-time. The performance of the system was assessed in a cadaveric upper extremity, and preliminary results of the effects of implant head position on kinematics were obtained. This system has provided new information that may enhance the selection and design of implants for reconstruction of the radial head, and lead to computer-assisted placement of these implants intraoperatively.
\end{abstract}

\section{Introduction}

The radial head allows for pronation-supination of the forearm. When the medial collateral ligament is injured, the radial head becomes the main stabilizer against lateral valgus forces. Similarly, when the interosseous membrane is damaged, the radial head becomes the main stabilizer against axial forces. In the case of a highly comminuted (unreconstructible) radial head fracture, there is often concomitant ligament injury, and therefore radial head arthroplasty is required to restore elbow and forearm function and stability

With the exception of a few bipolar designs [1;2] allowing the implant head to swivel relative to the stem, radial head implant designs are axisymmetric [3-7]. Both the bipolar and axisymmetric implants do not match the irregular morphology of the radial head; the radial head is ovoid in shape, and offset and angled with respect to the axis of the radial neck medullary canal [8-10]. 
When the stem of current radial head implant designs is placed in the canal of the radial neck, it is unlikely that the implant head will be in the same orientation as the native head. This is particularly relevant when rigid fixation of the stem is desired. Failure to restore the geometric characteristics of the native radial head may alter radiohumeral and radioulnar kinematics and joint forces. Abnormal forces may cause implant loosening, and capitellar cartilage wear.

This study focuses on the development of a computer-assisted method of positioning the radial head implant to better match the native geometry. Our specific objectives were: to (1) develop a computer-assisted implant alignment system for the radial head implant; and, (2) to develop an adjustable implant to investigate the effect of implant position on joint function in vitro.

\section{Methods}

Integral parts of this computer-assisted system included the electromagnetic tracking device (Flock of Birds, Ascension Technology, Burlington, VT), a specially designed modular and adjustable radial head implant (and its accessories), and custom-coded LabVIEW software (National Instruments, Austin, TX). In addition, the techniques used for quantifying radial head implant position, and testing of the system in a cadaveric specimen, are described.

\subsection{Tracking Device}

The Flock of Birds electromagnetic tracking device, which outputs the position of multiple receivers ( $\mathrm{R} 1, \mathrm{R} 2$, etc...) relative to the transmitter in six degrees of freedom (three rotations and three translations), was used. (Fig. 1).

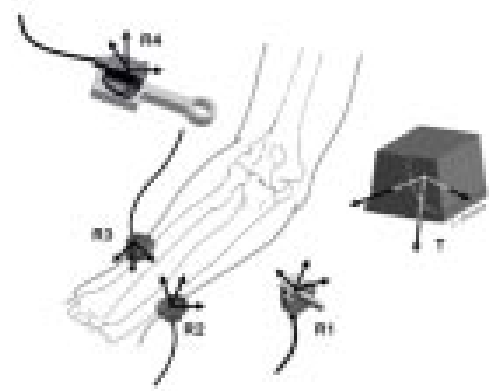

Fig. 1. Electromagnetic Tracking Device: R2 and R3 are rigidly fixed to the ulna and radius respectively. $\mathrm{R} 1$ is attached to a probe used to digitize bony landmarks. A fourth receiver (R4) is secured to the implantpositioning tool (described in Section 2.3).

\subsection{Custom Modular Implant}

A custom metallic radial head implant was developed (Fig. 2). The main features are an implant stem, an adjustable yoke, and an articulating shell surface. The adjustable yoke allows the implant head to be adjusted and locked in any position within an offset range of 0 to $4.5 \mathrm{~mm}$ and angular range of 0 to 20 degrees. Once the yoke is in the desired translation and rotation (offset and orientation), it is locked in place, and the articulating shell is secured to the yoke. 


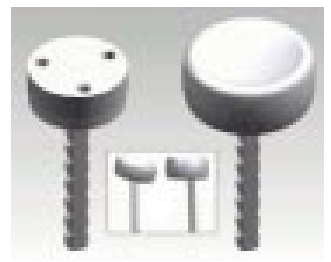

Fig. 2. Custom radial head implant: (Left) Implant with adjustable yoke shown. (Right) Articulating shell surface in place. (Inset) Example of head angle and offset positions.

\subsection{Implant-Positioning Tool}

The implant-positioning tool determines the location of the implant head relative to the stem and host (radial) bone. The tool head is a cylindrical section that engages (fits over) the adjustable yoke, thus enabling positioning (Fig. 3.a). The tool head also provides access to the clamping screws on the yoke for fixing the yoke in position (Fig. 3.c).
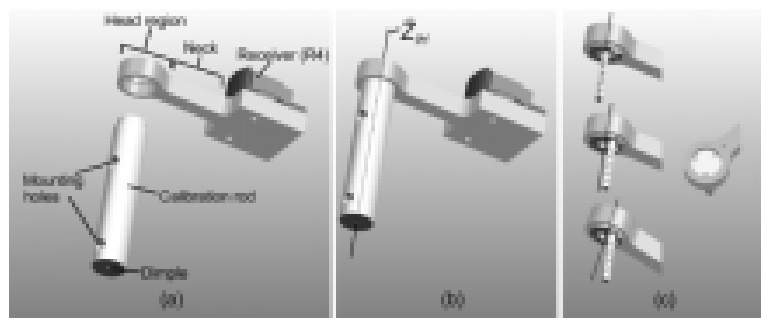

Fig. 3. Implant-positioning tool calibration: (a) The head of the tool is placed over a rigidly mounted cylindrical calibration rod. This cylinder has a dimple machined at the centre of each end. Using the tracking device's digitizing probe (R1) these dimples are digitized, and the position of these points relative to $\mathrm{R} 1$ recorded. (b) The vector connecting these two digitized points corresponds to the axis of the yoke $\left(\vec{Z}_{\mathrm{rH}}\right)$. (c) As the positioning tool is manipulated to change the implant head position, $\vec{Z}_{\mathrm{IH}}$ moves accordingly.

\subsection{Computer-Assisted Tracking Design and Development}

A custom LabVIEW program was used to calibrate the implant-positioning tool, establish the native head orientation, determine the axisymmetric implant coordinate system, and allow for the position of the adjustable implant to be tracked interactively. When digitizing points or contours of the native radial head relative to the transmitter, the LabVIEW program performs the necessary transformations in order to record and display the digitizations relative to any chosen receiver.

\subsubsection{Implant-Positioning Tool Calibration}

The implant-positioning tool was calibrated preoperatively. The procedure is described in Fig. 3. By determining the orientation of R4 relative to R3 (rigidly mounted on the radius), and considering the tool calibration, the translation and rotation of the inner head, $\vec{Z}_{\mathrm{IH}}$, can be visualized on-screen as the tool is manipulated. 


\subsubsection{Native Radial Head Coordinate System}

The radial head is exposed by performing a lateral epicondylar osteotomy to allow the radial head to be repeatedly accessed while restoring lateral ligament integrity during testing. The articulating dish, the rim of the dish, the circumferential edge of the radial head, a point on the lateral edge of the radial head (point $\mathrm{L}$ ) and a point in space proximal to the dish centre (point Z) are digitized (Fig. 4). A circle is fitted to the dish rim data points using a least squares-based algorithm and the centre of the circle (point $\mathrm{D}_{R}$ ) determined. A similar data reduction is performed on the articulating dish data points, and the centre point of this sphere-fit (point $S$ ) determined. A vector joining point $D_{R}$ and point $S$ is created and corresponds to the native radial head (dish) orientation $\left(\vec{Z}_{\mathrm{RH}}\right)$. This vector is essentially the line perpendicular to the plane of the dish rim. The deepest point in the dish (point $\mathrm{D}_{\mathrm{D}}$ ) is determined; this point is selected as the origin of the radial head coordinate system and can be used to match the implant to the native radial head height. Points $\mathrm{L}$ and $\mathrm{Z}$ are used as references to establish direction of the axes. The positive-x (medial-lateral) axis, $\vec{X}_{R H}$, goes through point $\mathrm{L}$. The $\mathrm{z}$ (proximal-distal) axis, $\overrightarrow{\mathrm{Z}}_{\mathrm{RH}}$, does not go through point $\mathrm{Z}$, although point $\mathrm{Z}$ is used to establish the positive-z direction. The positive-y direction, $\overrightarrow{\mathrm{Y}}_{\mathrm{RH}}$ is found using the cross product of the other two axes.

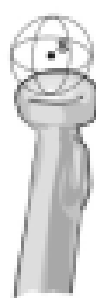

(a)

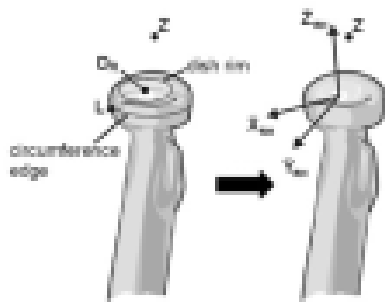

(b) (c)

Fig. 4. Creating the radial head coordinate system: (a) The articulating dish is digitized, and the centre of its sphere-fit (point $S$ ) determined. (b) A point on the lateral edge of the radial head was digitized (L), as well as the rim of the articulating dish. The rim was circle-fitted to find its centre point, $D_{R}$. (c) From these digitizations, the local radial head coordinate system was created.

\subsubsection{Initial Implant Positioning and Head Orientation Visualization}

Initially the implant head position is secured in its axisymmetric position using an alignment jig (not shown). This allowed the implant to mimic the geometry of many currently available axisymmetric radial head implants. Without computer assistance, the implant is positioned by an experienced orthopaedic surgeon and rigidly cemented into place using bone cement.

Once the tool is in place, the orientation of the tool, and therefore the implant head, is recorded with respect to the previously established radial head coordinate system. The orientation is represented graphically by the three-axes of the native head and $\vec{Z}_{\mathrm{IH}}$ of the implant head. The program also provides a numerical description of the orientation and offset (Section 2.4.3.1 \& 2.4.3.1). 
With the tool in place on the axisymmetrically oriented implant, the program records the orientation. For subsequent implant positions the program reports the implant head orientation with respect to both the native system and axisymmetric position.

\subsubsection{Implant Head Angle}

Two angles are used to describe overall angular position: $\alpha$, implant head tilt; and $\beta$, direction of tilt. For example, for a tilt of $10^{\circ}$ in the anterior direction, $\alpha$ would equal $10^{\circ}$ and $\beta$ would be $90^{\circ}$. $\beta$ (tilt direction) can range from $0^{\circ}$ to $360^{\circ}$, with the lateral, anterior, medial and posterior directions corresponding to $0^{\circ}, 90^{\circ}, 180^{\circ}$, and $270^{\circ}$ respectively.

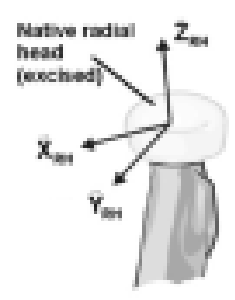

(a)

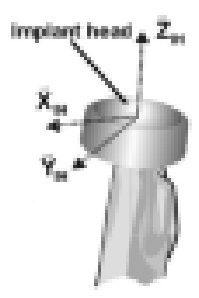

(b)

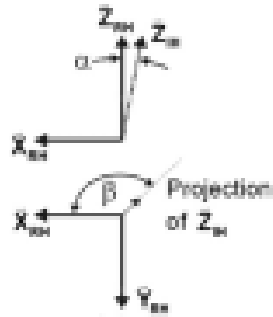

(c)

Fig. 5. Describing the implant head orientation using $\alpha$ and $\beta$ : (a) Shows the local native radial head coordinate system. (b) Shows the radial head implant coordinate system. Once the stem is cemented in place the head orientation can be altered. The axis of the implant head is $\vec{Z}_{\mathrm{IH}}$. (c) The orientation of $\vec{Z}_{\mathrm{IH}}$ (implant head) is described as two angles: $\alpha$, the angle that $\vec{Z}_{\mathrm{IH}}$ makes with $\vec{Z}_{\mathrm{RH}}$ (top); and $\beta$, the angle that the projection of $\vec{Z}_{\mathrm{IH}}$ on the $\mathrm{X}_{\mathrm{RH}}-\mathrm{Y}_{\mathrm{RH}}$ plane of the radial head coordinate system makes with $\overrightarrow{\mathrm{X}}_{\mathrm{RH}}$ (bottom).

\subsubsection{Implant Head Offset}

The implant head offset can be described in two ways, with respect to the native radial head system or with respect to the axisymmetric implant position, as described in Fig. 6.

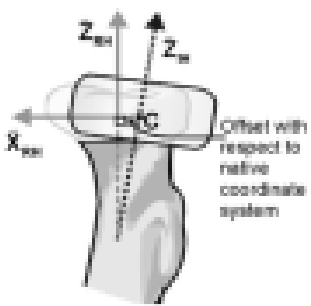

(a)

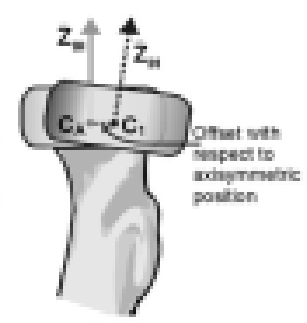

(b)

Fig. 6. Determining implant head offset: (a) When describing the offset with respect to the native coordinate system, it is the distance along a vector perpendicular to $\vec{Z}_{\mathrm{RH}}$ from $\vec{Z}_{\mathrm{RH}}$ to the implant head centre, C. (b) When describing the offset with respect to the axisymmetric position, it is distance from the centre of the implant head, when in the axisymmetric position, $\mathrm{C}_{\mathrm{A}}$, to the centre of the implant head in its new position, $\mathrm{C}_{1}$. 


\subsection{Cadaver-Based Trials}

Function of the system was tested in one cadaveric specimen using procedures established in our laboratory $[6 ; 11 ; 12]$. The electromagnetic tracking system receivers were rigidly secured to the bones of the forearm (see Section 2.1). In addition to implant positioning, the tracking system allowed the measurement of kinematics from active and passive simulated elbow and forearm motion.

The implant was cemented in place as described in Section 2.4.3 and subsequently adjusted to seven different head translations and/or rotations.

\section{Results}

While adjusting the implant head translation and orientation, real-time graphical representation of head orientation relative to the native radial head coordinate system appeared on-screen. A number of digitizations were displayed to aid in placement of the yoke, including the edge circumference, and points corresponding to the centre of rotation of the implant head (point $C$ ) and to the centermost/deepest point of the dish surface (point $\left.\mathrm{D}_{\mathrm{Di}}\right)$ on the tool axis $\left(\overrightarrow{\mathrm{Z}}_{\mathrm{IH}}\right)$. The graphical display of two of the implant head positions, "dish match" and "edge match" is shown in Figs. $7 \& 8$ respectively.

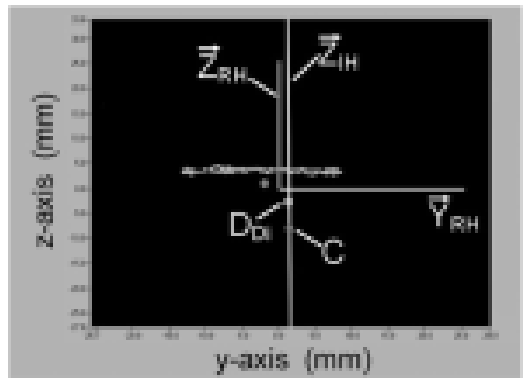

(a)

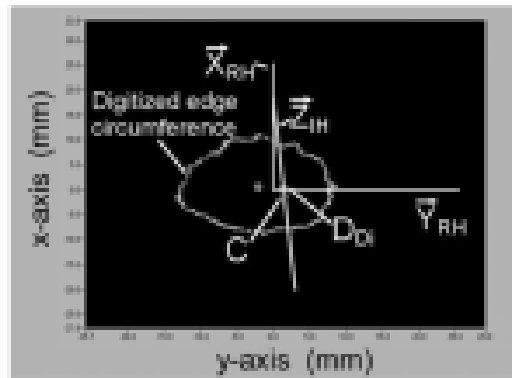

(b)

Fig. 7. Matching the native dish orientation: The tool axis $\left(\vec{Z}_{I H}\right)$ and the $\mathrm{Z}$-axis of the radial head coordinate system $\left(\vec{Z}_{R H}\right)$ are aligned as closely as possible. (a) Shows the $Y_{R H}-Z_{R H}$ view, the implant centre of rotation, $\mathrm{C}$, and the deepest dish point, $\mathrm{D}_{\mathrm{Di}}$, are also shown along the tool axis. (b) Shows the $\mathrm{Y}_{\mathrm{RH}}-\mathrm{X}_{\mathrm{RH}}$ view. The edge circumference digitization is visible in both views.

\section{Discussion}

This is the first reported use of computer-assisted arthroplasty at the elbow. This system is currently for use in vitro where the intact radial head can be digitized prior to arthroplasty. Clinically this is not the case, however the normal radial head 
morphology of the intact contralateral elbow could be obtained from imaging with Computed Tomography. This may allow clinical computer-assisted methods to be developed similar to those employed in this study

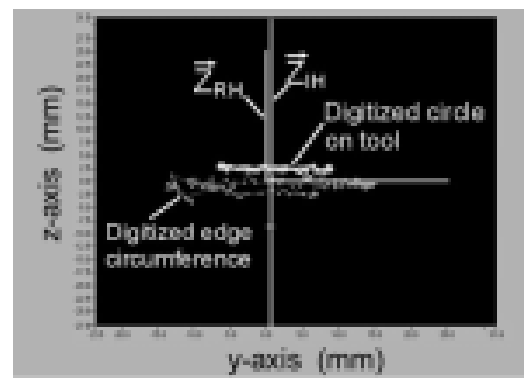

(b)

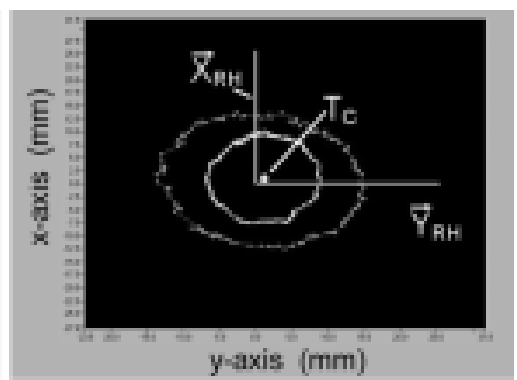

(a)

Fig. 8. Matching the edge of the native head: The tool circle and the circumference of the edge of the radial head were aligned such that they were concentric and parallel in all three views. The centre of the tool circle, $T_{C}$, was also used during alignment. (a) Shows the $Y_{R H}-Z_{R H}$ view. (b) Shows the $\mathrm{Y}_{\mathrm{RH}}-\mathrm{X}_{\mathrm{RH}}$ view.

In its current form, the system described will allow the effect of head translation and rotation to be studied. With modifications to both the computerized guidance system and the prototype implant other factors like implant head height and shape (e.g. circular versus ovoid) can be investigated. This system should enhance the design and surgical placement of implants for reconstruction of the radial head.

\section{References}

1. Judet T, Garreau dL, Piriou P, Charnley G: A floating prosthesis for radial-head fractures. J Bone Joint Surg Br 78:244-249, 1996

2. Pomianowski S, Morrey BF, Neale PG, Park MJ, O'Driscoll SW, An KN: Contribution of monoblock and bipolar radial head prostheses to valgus stability of the elbow. J Bone Joint Surg Am 83-A:1829-1834, 2001

3. Harrington IJ, Tountas AA: Replacement of the radial head in the treatment of unstable elbow fractures. Injury 12:405-412, 1981

4. Harrington IJ, Sekyi-Otu A, Barrington TW, Evans DC, Tuli V: The functional outcome with metallic radial head implants in the treatment of unstable elbow fractures: a long-term review. J Trauma 50:46-52, 2001

5. Knight DJ, Rymaszewski LA, Amis AA, Miller JH: Primary replacement of the fractured radial head with a metal prosthesis. Journal of Bone and Joint Surgery 75B:572-576, 1993

6. King GJ, Zarzour ZD, Rath DA, Dunning CE, Patterson SD, Johnson JA: Metallic radial head arthroplasty improves valgus stability of the elbow. Clinical Orthopaedics and Related Research 368:114-125, 1999

7. Moro JK, Werier J, MacDermid JC, Patterson SD, King GJW: Arthroplasty with a metal radial head for unreconstructible fractures of the radial head. Journal of Bone and Joint Surgery 83A:1201-1211, 2001

8. Cone RO, Szabo R, Resnick D, Gelberman R, Taleisnik J, Gilula LA: Computed tomography of the normal radioulnar joints. Invest Radiol 18:541-545, 1983 
9. King GJ, Zarzour ZD, Patterson SD, Johnson JA: An anthropometric study of the radial head: implications in the design of a prosthesis. Journal of Arthroplasty 16:112-116, 2001

10. Stacpoole RA. Biomechanical factors related to the fixation and kinematics of radial head arthroplasty. 2002. University of Western Ontario.

11. Johnson JA, Rath DA, Dunning CE, Roth SE, King GJ: Simulation of elbow and forearm motion in vitro using a load controlled testing apparatus. Journal of Biomechanics 33:635639,2000

12. Dunning CE, Duck TR, King GJ, Johnson JA: Simulated active control produces repeatable motion pathways of the elbow in an in vitro testing system. J Biomech 34:1039-1048, 2001 